\title{
Quaternion Demonstration of the Theorem of Moments in Rigid Dynamics.
}

\author{
By Dr Cargill G. Knotr.
}

(Read 12th February 1915. Received 12th March 1915.)

In a recent number of the American Mathematical Monthly (December 1914), Professor E. V. Huntington calls attention to the inconclusive, and in some cases erroneous, discussion of the uniplanar motion of a rigid body as it is presented in the more elementary books on mechanics. In the simpler problems considered the solution is usually found by use of the rule that the rate of change of moment of momentum about some convenient point is equal to the torque or turning moment about the same point. In many cases there is no difficulty choosing this convenient point; but apparently there is confusion as to the points which can be legitimately chosen.

Professor Huntington shows in a simple way that the point about which moments may be taken must satisfy at least one of four conditions. The investigation is limited to cases of uniplanar motion. The problem in its completely general tri-dimensional form is readily solved by quaternion analysis.

The following investigation is very similar to the discussion I give in the Third Edition of Kelland and Tait's Introduction to Quaternions (\$72, p. 160). It is, however, more general.

With reference to any fixed origin, let $\rho$ be the vector position of the mass-element $m$, and let $\beta$ be the force acting on it. By Lex. II.

$$
\left.\begin{array}{rl}
m \ddot{\rho} & =\beta \text { for each mass } \\
\Sigma m \ddot{\rho} & =\Sigma \beta \text { for the whole system }
\end{array}\right\}
$$

Here $\Sigma \beta$ includes only the applied external forces, the internal forces cancelling one another in accordance with Lex. III. 
Multiplying each single equation by the corresponding $\rho$ and summing for the whole system, we get

$$
\Sigma m \rho \ddot{\rho}=\Sigma \rho \beta
$$

The scalar part of this gives the virial equation, namely, $\Sigma S m \rho \ddot{\rho}=\Sigma S \rho \beta$, and in it $\beta$ includes the internal forces. The vector part, namely, $\Sigma V_{m} \ddot{\rho}=\Sigma V_{\rho} \beta$ gives the theorem of moments; and in it $\beta$ does not include the internal forces, in virtue of Lex. III.

In equations (1) and (2) put

$$
\rho=\omega+\sigma, \ddot{\rho}=\ddot{\omega}+\ddot{\sigma}
$$

where $\omega$ is the vector position of some definite point, at rest or in motion, and $\sigma$ is the vector position relative to this point of the mass element at $\rho$. We find, since $\omega$ and $\ddot{\omega}$ may be placed outside the summation symbol,

$$
\begin{aligned}
& \ddot{\omega} \Sigma m+\Sigma m \ddot{\sigma}=\Sigma \beta \ldots \ldots \\
& \omega \ddot{\omega} \Sigma m+\omega \Sigma m \ddot{\sigma}+(\Sigma m \sigma) \ddot{\omega}+\Sigma m \sigma \ddot{\sigma}=\omega \Sigma \beta+\Sigma \sigma \beta
\end{aligned}
$$

But, multiplying $\left(1^{\prime}\right)$ by $\omega$ we get

$$
\ddot{\omega} \Sigma m+\omega \Sigma m \ddot{\sigma}=\omega \Sigma \beta
$$

which, subtracted from $\left(2^{\prime}\right)$, gives

$$
(\Sigma m \sigma) \ddot{\omega}+\Sigma m \sigma \ddot{\sigma}=\Sigma \sigma \beta
$$

Now $\Sigma m \sigma=\mu \Sigma m$, where $\mu$ is the vector of the centre of mass. Hence, taking the vector part of (3) we find

$$
\nabla \mu \ddot{\omega} \Sigma m+\Sigma V m \sigma \ddot{\sigma}=\Sigma V \sigma \beta
$$

The question then is, What conditions must be satisfied in order that the point $\omega$ may be legitimately chosen as a pivot with regard to which the theorem of moments may hold? that is, when

$$
\Sigma V_{m} \ddot{\sigma}=\Sigma \nabla \sigma \beta \text {. }
$$

Obviously the conditions are given by the equation

$$
\nabla \mu \ddot{\omega}=0 \text {. }
$$


Now the vanishing of this vector produced may happen in one of three ways :-

(1) $\mu=0$, or the pivot point is the centre of mass.

(2) $\ddot{\omega}=0$, or the pivot point has no acceleration; that is, it may be a point moving with constant velocity (uniform speed in a straight line) or a point completely at rest.

(3) $\ddot{\omega}$ parallel to $\mu$, or the point's acceleration must be directed through the centre of mass.

Only those points which satisfy one at least of these conditions can be used as pivot points in the application of the theorem of moments.

The conditions may be expressed even more concisely under two headings; for it is obvious that whatever may be the acceleration of the centre of mass, that acceleration must pass through its own position. Hence,

All points which can be used as pivot points in the theorem of moments must have their acceleration either zero or directed through the position of the centre of mass. 